\title{
Synthesis of
}

Tetrahydronaphthyridines from Aldehydes and HARP Reagents via Radical Pictet-Spengler

\section{Reactions}

\section{Journal Article}

Author(s):

Jackl, Moritz K.; Kreituss, Imants; Bode, Jeffrey W.

Publication date:

2016-04-15

Permanent link:

https://doi.org/10.3929/ethz-b-000260485

Rights / license:

In Copyright - Non-Commercial Use Permitted

Originally published in:

Organic Letters 18(8), https://doi.org/10.1021/acs.orglett.6b00523

Funding acknowledgement:

306793 - Catalytic asymmetric synthesis of amines and amides (EC) 


\title{
Synthesis of Tetrahydronaphthyridines from Aldehydes and HARP Reagents via Radical Pictet-Spengler Reactions
}

\author{
Moritz K. Jack1, ${ }^{\S}$ Imants Kreituss ${ }^{\S}$ and Jeffrey W. Bode* \\ Laboratorium für Organische Chemie, Department of Chemistry and Applied Biosciences, \\ ETH-Zürich, 8093 Zürich, Switzerland \\ Supporting Information Placeholder
}

\begin{abstract}
The combination of aldehydes with newly designed HARP (Halogen Amine Radical Protocol) reagents gives access to $\alpha$-substituted tetrahydronaphthyridines. By using different HARP reagents, various regioisomeric structures can be prepared in a single operation. These products, which are of high value in medicinal chemistry, are formed in a predictable manner via a formal Pictet-Spengler reaction of electron poor pyridines that would not participate in the corresponding polar reactions.
\end{abstract}

Fused aromatic/saturated N-heterocycles are important scaffolds for biologically active pharmaceuticals and natural products. ${ }^{\mathbf{1 1 2}}$ While many widely used examples - including tetrahydroisoquinolines and $\beta$-tetrahydrocarbinols - can be accessed by established processes such as Pictet-Spengler ${ }^{3}$ or Bischler-Napieralski ${ }^{4}$ reactions, these approaches are not well suited for the preparation of electron-deficient systems such as tetrahydronaphthyridines. ${ }^{5}$ The poor synthetic access to such compounds is exemplified by recent work from Amgen, where the target molecule synthesis relied on introduction of the substituents in the very first step of a long synthetic sequence. ${ }^{6}$ While such an approach is suitable once a lead compound is identified, it is not appealing in early stages where library syntheses and structure activity relationship studies are the primary goal.

As part of a program aimed at establishing predictable, cross-coupling approaches to saturated N-heterocycles from widely available components, our group has developed SnAP (stannyl amine protocol) reagents, ${ }^{7}$ which enable the single step synthesis of diverse, saturated $\mathrm{N}$-heterocycles from aldehydes (Scheme 1). We sought to extend this concept to the preparation of partially saturated bicyclic heterocycles, beginning with otherwise difficult to access tetrahydronaphthyridines in various regioisomeric forms. In this communication, we document our successful development of a new class of reagents, HARP (Halogen Amine Radical Protocol) reagents, and conditions suitable for this cross-coupling. Although the mechanism involves radical additions to imines - the key step of the SnAP chemistry - our solution to these product classes relies on a different radical source and reaction conditions. The new approach not only allows the assembly of fused saturated/aromatic $\mathrm{N}$-heterocycle scaffolds, it also delivers the expected products in a predictable manner, overcoming the inherent regioselectivity problems associated with the PictetSpengler or Bischler-Napieralski reactions.
Scheme 1. SnAP and HARP reagents

Prior work: SnAP reagents for saturated N-heterocycle synthesis

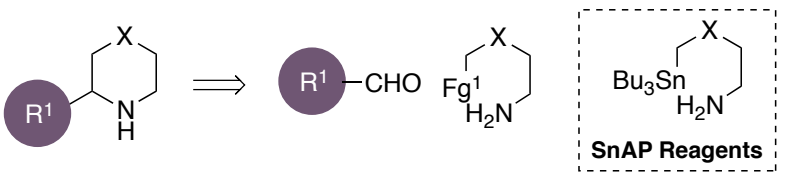

This work: HARP reagents for fused saturated/aromatic $\mathrm{N}$-heterocycles<smiles>NCCc1ccccc1NC(=O)c1ccc(C=Cc2ccccc2)cc1</smiles>

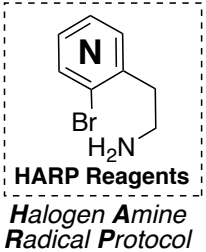

Selected examples:<smiles>c1ccc(CCC2NCCc3cccnc32)cc1</smiles><smiles>c1cncc(C2NCCc3ccncc32)c1</smiles>

Although aryl tin reagents could serve as the source of radicals ( $\mathrm{Fg}^{1}$ in Scheme 1), we sought to identify a starting material that was both easier to prepare and avoided the use of organostannes. We reasoned that the aromatic halides should be sufficiently stable and would generate the corresponding $\mathrm{sp}^{2}$ centered radical upon treatment with a radical initiator. ${ }^{\mathbf{8}}$ We therefore began our investigations with the preparation of 2bromopyridine reagent 3 from the corresponding aldehyde on a multigram scale (Scheme 2). ${ }^{9}$ 
Scheme 2. Synthesis of HARP Reagents

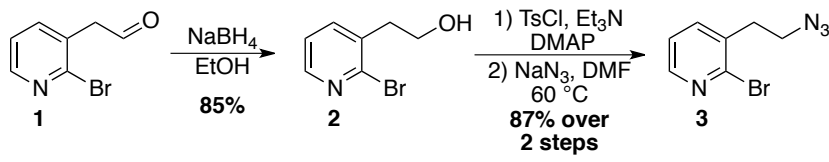

The azide could be reduced to the amine, but we found it to be air and moisture insensitive and elected to use this reagent for imine formation. Staudinger reduction with polymer bound triphenylphosphine followed by an aza-Wittig reaction with the corresponding aldehyde cleanly gave the requisite imines, which were isolated by simple filtration from the resin. ${ }^{7 \mathrm{~d}}$ This approach also allayed fears of intramolecular cyclization of the amine to form an indoline. Using 4trifluoromethylbenzaldehyde as a substrate for reaction optimization, we tested several conditions for the radical generation and cyclization. The use of $\mathrm{Et}_{3} \mathrm{~B} / \mathrm{O}_{2}{ }^{10}$ or $\mathrm{AIBN} / \mathrm{Bu}_{3} \mathrm{SnH}^{11}$ led to complex product mixtures and iridium ${ }^{\mathbf{1 2}}$ or organocatalytic photoredox systems did not exhibit the desired reactivity. We were, however, pleased to find that catalytic amount of AIBN in combination with (TMS) ${ }_{3} \mathrm{SiH}^{\mathbf{1 3}}$ provided the cyclized product, which was isolated in $84 \%$ yield after column chromatography. Encouraged by these results we continued the investigation of the substrate scope. The imine formation and cyclization proceeded equally well with both electron poor and electron rich aldehydes and tolerated heterocyclic and orthosubstituted substrates. Products derived from aliphatic aldehydes could be isolated in acceptable yields. Surprisingly, aromatic aldehydes containing halogen atoms $(-\mathrm{Cl}$ and $-\mathrm{Br})$ were suitable substrates and we did not observe dehalogenation during the cyclization. We attribute this to the kinetically favored generation of the more stable 2-pyridyl radical. ${ }^{\mathbf{1 4}}$

Given the key role of the 2-pyridyl halide for efficient radical generation, it was unclear if our concept could be extended to the construction of regioisomeric tetrahydronaphthyridines. The necessary reagents - also as the azides - could be prepared by straightforward routes (see Supporting Information) and imine formation using the phosphine resin proceeded equally well. As anticipated, the cyclization conditions employed for the first reagent (3) gave only trace amounts of the desired product. Lewis acidic additives such as $\mathrm{BF}_{3} \bullet \mathrm{Et}_{2} \mathrm{O}^{15}$ or the use of Brønsted acidic solvents such as HFIP ${ }^{\mathbf{1 6}}$ - which is crucial for cyclizations using SnAP reagents - led to the decomposition of the imine intermediate.

By increasing the amount of AIBN we could isolate the cyclic amines in their $\mathrm{N}$-unprotected form (Scheme 4). The yields are considerably lower than for the 2-pyridil halogen reagents - and therefore a subject for further investigation but even at the current state of development HARP reagents provide an attractive route to these products. We attribute the lower yields and somewhat more restricted substrate scope to the reduced stability of the aryl radical, which does not benefit from stabilization from an adjacent heteroatom. ${ }^{\mathbf{1 7}}$

This methodology serves as an expansion of our previous established SnAP reagent based synthesis of saturated Nheterocycles. Applications of this strategy to assemble other electron poor heterocyclic substrates are on the way in our laboratories.
Scheme 3. Synthesis of 1,7-Tetrahydronaphthyridines

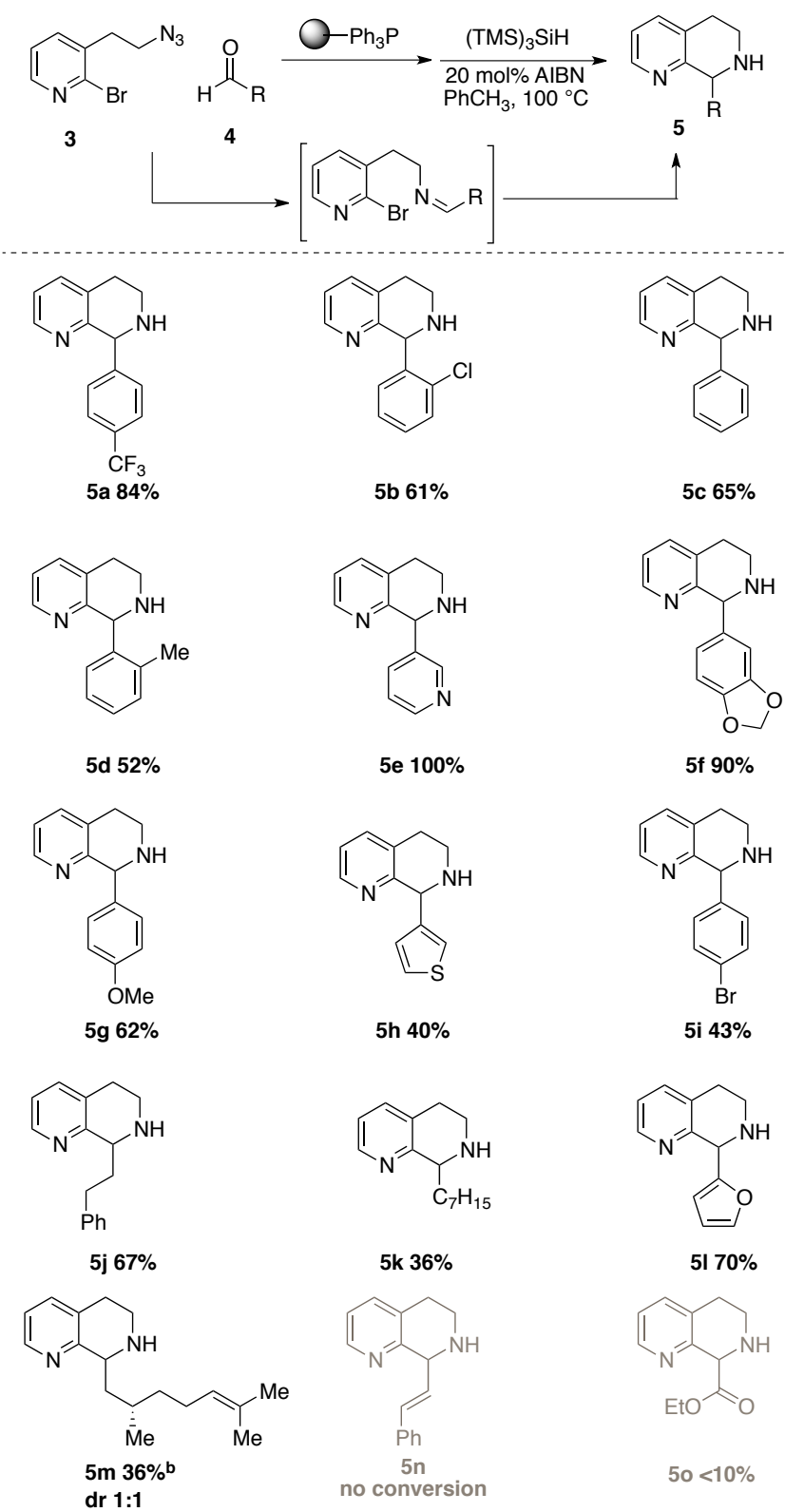

${ }^{a}$ Reaction condition for imine formation: azido reagent $(1.00$ equiv), aldehyde (0.95-1.00 equiv), polymer-bound triphenylphosphine (2.0 equiv), THF, $55^{\circ} \mathrm{C}, 12 \mathrm{~h}$. Condition for cyclization: imine (1.0 equiv), $\mathrm{AIBN}$ ( 0.2 equiv), (TMS $)_{3} \mathrm{SiH}$ (1.5-2.0 equiv), toluene, $100{ }^{\circ} \mathrm{C}$. Yields refer to isolated amine products after purification by column chromatography. ${ }^{b}$ Alongside product $\mathbf{5 m}$ the corespoding cyclic imine was obtained in $24 \%$ yield (see Supporting Information)

In summary, we have developed a new class of reagents to access $\alpha$-substituted tetrahydronaphthyridines and their regioisomers via a formal Pictet-Spengler reaction in a predictable manner. The requisite reagents are air and moisture stable and can be prepared on a multigram scale in a few synthetic steps from readily available starting materials. Applications of this strategy to assemble other electron poor heterocyclic substrates are on the way in our laboratories. 
Scheme 4. 1,6- and 2,7- Tetrahydronaphthyridine Synthesis

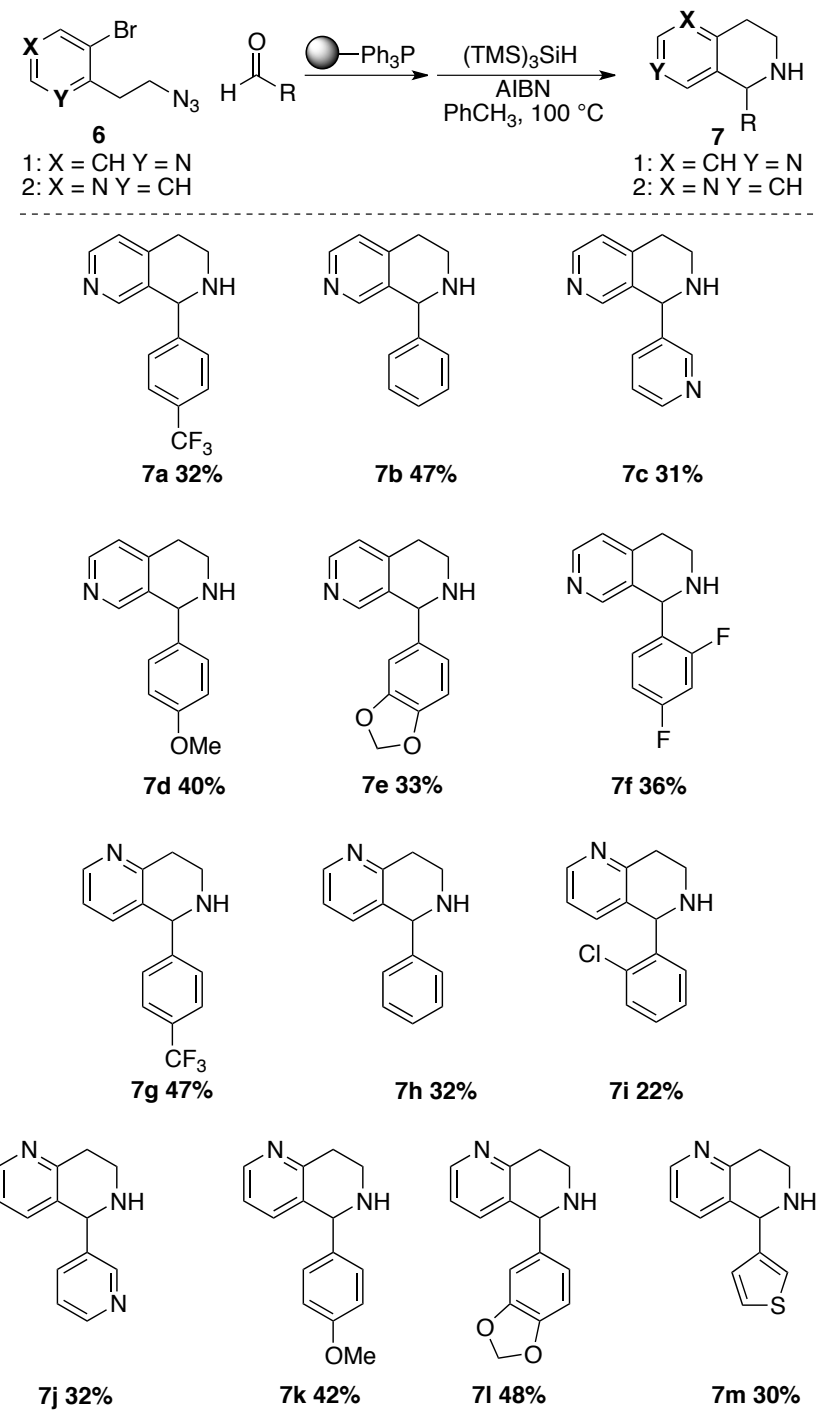

${ }^{a}$ Reaction condition for imine formation: azido reagent $(1.00$ equiv), aldehyde (0.95-1.00 equiv), polymer-bound triphenylphosphine (2.0 equiv), THF $55^{\circ} \mathrm{C}, 12 \mathrm{~h}$. Condition for cyclization: imine (1.0 equiv), $\mathrm{AIBN}(0.2-1.0 \text { equiv), (TMS })_{3} \mathrm{SiH}$ (1.5-2.0 equiv), toluene $100{ }^{\circ} \mathrm{C}$. Yields refer to isolated amine products after purification by column chromatography.

\section{ASSOCIATED CONTENT}

Supporting Information. Experimental procedures and analytical data of all new compounds. This material is available free of charge via the Internet at http://pubs.acs.org.

\section{AUTHOR INFORMATION}

\section{Corresponding Author}

bode@org.chem.ethz.ch

Author Contribution

$\S$ These authors contributed equally to this work.

\section{Funding Sources}

This work was supported by the European Research Council (ERC Starting Grant No. 306793 - CASAA) and the Swiss Federal Commission for Technology and Innovation (CTI 15523.1).

\section{ACKNOWLEDGMENT}

We would like to thank Michael U. Luescher and Shen-Ying Hsieh (ETH) for helpful discussions.

\section{REFERENCES}

(1) a) Taylor, R. D.; MacCoss, M.; Lawson, A. G. J. Med. Chem. 2014, 57, 5845 - 5859. b) Vitaku, E.; Smith, D. T.; Njardarson, J. T. J. Med. Chem. 2014, 57, 10257 - 10274. c) Xiong, B.; Li, Y.; Lv, W.; Tan, Z.; Jiang, H.; Zhang, M. Org. Lett. 2015, 17, 4054 - 4057. d) Basarab, G. S.; Galullo, V.; DeGrace, N.; Hauck, S.; Joubran, C.; Wesolowski, S. S. Org. Lett. 2014, 16, 6456 - 6459. e) Palucki, M.; Hughes, D. L.; Yasuda, N.; Yang, C.; Reider, P. J. Tetrahedron. Lett. 2001, 42, 6811 - 6814. f) Haussenether, T.; Troschutz, R. J. Heterocycl. Chem. 2004, 41, $857-865$.

(2) Murray, C. W.; Rees, D. C. Angew. Chem. Int. Ed. 2016, 55, $488-$ 492.

(3) a) Pictet, A.; Spengler, T. Berichte der Deutschen Chemischen Gesellschaft 1911, 44, 2030 - 2036. b) Raheem, I. T.; Thiara, P. S.; Peterson, E. A.; Jacobsen, E. N. J. Am. Chem. Soc. 2007, 129, 13404 -13405 .

(4) Fodor, G.; Nagubandi, S. Tetrahedron 1980, 36, 1279 - 1300.

(5) Davis, F. A.; Melamed, J. Y.; Sharik. S. S. J. Org. Chem. 2006, $71,8761-8766$.

(6) Horne, D. B.; Tamayo, N. A.; Bartberger, M. D.; Bo, Y.; Clarine, J.; Davis, C. D.; Gore, V. K.; Kaller, M. R.; Lehto, S. G.; Ma, V. V.; Nishimura, N.; Nguyen, T. T.; Tang, P.; Wang, W.; Youngblood, B. D.; Zhang, M.; Gavva, N. R.; Monenschein, H.; Norman, M. H. J. Med. Chem. 2014, 57, 2989 - 3004.

(7) a)Vo, C. V.; Mikutis, G.; Bode, J. W. Angew. Chem. Int. Ed. 2013, 52, 1705 - 1708. b) Vo. C. V.; Luescher, M. U.; Bode, J. W.; Nature Chem. 2014, 6, 310 - 314. c) Luescher, M. U.; Vo, C. V.; Bode, J. W. Org. Lett. 2014, 16, 1236 - 1239. d) Siau, W-Y.; Bode, J. W. J. Am. Chem. Soc. 2014, 136, 17726 - 17729. e) Luescher, M. U.; Bode, J. W. Angew. Chem. Int. Ed. 2015, 54, 10884 - 10888. f) Geoghegan, K.; Bode J. W. Org. Lett. 2015, 17, 193401937.

(8) Studer, A.; Curran, D. P. Angew. Chem. Int. Ed. 2016, 55, $58-$ 102 .

(9) Heidelbaugh, T.; Chow, K.; Sinha, S.; Nguyen, P.; Fang, W.; Li, L.; Taekuchi, J.; Bhat, S. Allergan Inc Patent WO2009091760 (A1) 2009

(10) Renaud, P.; Beauseigneur, A.; Brecht-Forster, A.; Becattini, B.; Darmency, V.; Kandhasamy, S.; Montermini, F.; Ollivier, C.; Panchaud, P.; Pozzi, D.; Scanlan, E. M.; Schaffner, A-P.; Weber, V. Pure Appl. Chem. 2007, 79, 223 - 233.

(11) $\mathrm{Bu}_{3} \mathrm{SnH} / \mathrm{AIBN}$ combination has been reported for intramolecular cyclizations on imines a) Tomaszewski, M. J.; Warkentin J. Tetrahedron. Lett. 1992, 33, 2123 - 2126. b) Orito, K.; Uchiito, S.; Satoh, Y.; Tatsuzawa, T.; Harada, R.; Tokuda, M. Org. Lett. 1999, 2, 307 - 310. c) Johnston, J. N.; Plotkin, M. A.; Viswanathan, R.; Prabhakaran, E. N. Org. Lett. 2001, 3, 1009 - 1011. d) Viswanathan, R.; Prabhakaran, E. N.; Plotkin, M.; Johnston, J. N. J. Am. Chem. Soc. 2003, 125, 163 - 168. e) Viswanathan, R.; Mutnick, D.; Johnston, J. N. J. Am. Chem Soc. 2003, 125, $7266-7271$.

(12) Liu, D.; Liu, C.; Lei, A. Chem. Asian J. 2015, 10, 2040 - 2054

(13) a) Chatgilialogu C.; Timokhin, V. I. Silanes as Reducing Agents in Radical Chemistry in Encyclopedia of Radicals in Chemistry, Biology and Materials John Wiley \& Sons, 2012, DOI: 10.1002/9781119953678.rad074. b) Chatgilialoglu, C.; Lalevee, J. Molecules 2012, 17, 527 - 555 .

(14) Lucas, M.; Minor, J.; Zhang, J.; Brazier, C. J. Phys. Chem. A 2013, 117, 12138 - 12145 .

(15) Chen, Q.; Mollat du Jourdin, X.; Knochel, P. J. Am. Chem. Soc. 2013, 135, 4958 - 4961.b) b) Aubrecht, K. B.; Winemiller, M. D.; Collum, D. B. J. Am. Chem. Soc. 2000, 122, $11084-11089$.

(16) a) Eberson, L.; Hartshorn, M. P.; Persson, O.; Radner, F. Chem. Commun. 1996, 2105 - 2112. b) Berkessel, A.; Adrio, J. A.; Huttenhain, D.; Neudorfl, J. J. Am. Chem. Soc. 2006, 128, 8421 8426.

(17) Kikuchi, O.; Hondo, Y.; Morihashi, K.; Nakayama, M. Bull. Chem. Soc. Jpn. 1988, $291-292$. 
Insert Table of Contents artwork here

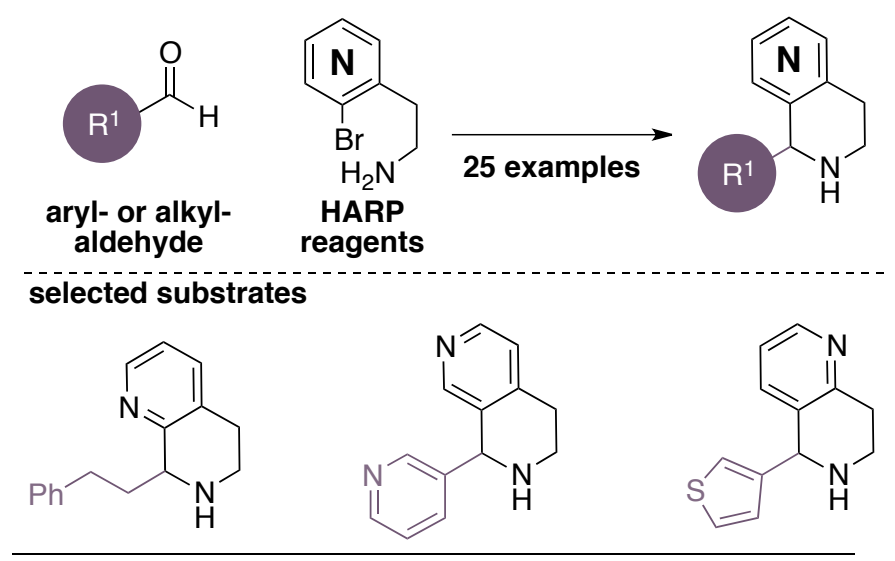


${ }^{1}$ a) Taylor, R. D.; MacCoss, M.; Lawson, A. G. J. Med. Chem. 2014, 57, 5845 - 5859. b) Vitaku, E.; Smith, D. T.; Njardarson, J. T. J. Med. Chem. 2014, 57, 10257 - 10274. c) Xiong, B.; Li, Y.; Lv, W.; Tan, Z.; Jiang, H.; Zhang, M. Org. Lett. 2015, 17, 4054 - 4057. d) Basarab, G. S.; Galullo, V.; DeGrace, N.; Hauck, S.; Joubran, C.; Wesolowski, S. S. Org. Lett. 2014, 16, 6456 - 6459. e) Palucki, M.; Hughes, D. L.; Yasuda, N.; Yang, C.; Reider, P. J. Tet. Lett. 2001, 42, 6811 - 6814. f) Haussenether, T.; Troschutz, R. J. Heterocyclic Chem. 2004, 41, 857 - 865.

${ }^{2}$ Murray, C. W.; Rees, D. C. Angew. Chem. Int. Ed. 2015, 54, DOI: 10.1002/anie.201506783

${ }^{3}$ a) Pictet, A.; Spengler, T. Berichte der Deutschen Chemischen Gesellschaft 1911, 44, 2030 - 2036. b) Raheem, I. T.; Thiara, P. S.; Peterson, E. A.; Jacobsen, E. N. J. Am. Chem. Soc. 2007, 129, $13404-$ 13405.

${ }^{4}$ Fodor, G.; Nagubandi, S. Tetrahedron 1980, 36, $1279-1300$.

${ }^{5}$ Davis, F. A.; Melamed, J. Y.; Sharik. S. S. J. Org. Chem. 2006, 71, $8761-8766$.

${ }^{6}$ Horne, D. B.; Tamayo, N. A.; Bartberger, M. D.; Bo, Y.; Clarine, J.; Davis, C. D.; Gore, V. K.; Kaller, M. R.; Lehto, S. G.; Ma, V. V.; Nishimura, N.; Nguyen, T. T.; Tang, P.; Wang, W.; Youngblood, B. D.; Zhang, M.; Gavva, N. R.; Monenschein, H.; Norman, M. H. J. Med. Chem. 2014, 57, 2989 - 3004.

${ }^{7}$ a)Vo, C. V.; Mikutis, G.; Bode, J. W. Angew. Chem. Int. Ed. 2013, 52, 1705 - 1708. b) Vo. C. V.; Luescher, M. U.; Bode, J. W.; Nature Chem. 2014, 6, 310 - 314. c) Luescher, M. U.; Vo, C. V.; Bode, J. W. Org. Lett. 2014, 16, 1236 - 1239. d) Siau, W-Y.; Bode, J. W. J. Am. Chem. Soc. 2014, 136, 17726 17729. e) Luescher, M. U.; Bode, J. W. Angew. Chem. Int. Ed. 2015, 54, 10884 - 10888. f) K. Geoghegan, J. W. Bode, Org. Lett. 2015, 17, 193401937.

${ }^{8}$ Studer, A.; Curran, D. P. Angew. Chem. Int. Ed. 2015, 54, DOI: 10.1002/anie.201505090.

${ }^{9}$ Heidelbaugh, T.; Chow, K.; Sinha, S.; Nguyen, P.; Fang, W.; Li, L.; Taekuchi, J.; Bhat, S. Allergan Inc Patent WO2009091760 (A1) 2009

${ }^{10}$ Renaud, P.; Beauseigneur, A.; Brecht-Forster, A.; Becattini, B.; Darmency, V.; Kandhasamy, S.; Montermini, F.; Ollivier, C.; Panchaud, P.; Pozzi, D.; Scanlan, E. M.; Schaffner, A-P.; Weber, V. Pure Appl. Chem. 2007, 79, $223-233$.

${ }_{11} \mathrm{Bu}_{3} \mathrm{SnH} / \mathrm{AIBN}$ combination has been reported for intramolecular cyclizations on imines a) Tomaszewski, M. J.; Warkentin J. Tett. Lett. 1992, 33, 2123 - 2126. b)Orito, K.; Uchiito, S.; Satoh, Y.; Tatsuzawa, T.; Harada, R.; Tokuda, M. Org. Lett. 1999, 2, 307 - 310. c) Johnston, J. N.; Plotkin, M. A.; Viswanathan, R.; Prabhakaran, E. N. Org. Lett. 2001, 3, 1009 - 1011. d) Viswanathan, R.; Prabhakaran, E. N.; Plotkin, M.; Johnston, J. N. J. Am. Chem. Soc. 2003, 125, 163 - 168. e) Viswanathan, R.; Mutnick, D.; Johnston, J. N. J. Am. Chem Soc. 2003, 125, 7266 - 7271.

${ }^{12}$ Liu, D.; Liu, C.; Lei, A. Chem. Asian J. 2015, DOI: 10.1002/asia.201500326.

${ }^{13}$ a) Chatgilialogu C.; Timokhin, V. I. Silanes as Reducing Agents in Radical Chemistry in Encyclopedia of Radicals in Chemistry, Biology and Materials John Wiley \& Sons, 2012, DOI: 10.1002/9780470971253.rad074. b) Chatgilialoglu, C.; Lalevee, J. Molecules 2012, 17, 527 - 555.

${ }^{14}$ Lucas, M.; Minor, J.; Zhang, J.; Brazier, C. J. Phys. Chem. A 2013, 117, 12138.

${ }^{15}$ Chen, Q.; Mollat du Jourdin, X.; Knochel, P. J. Am. Chem. Soc. 2013, 135, 4958 - 4961.b) b) Aubrecht, K. B.; Winemiller, M. D.; Collum, D. B. J. Am. Chem. Soc. 2000, 122, $11084-11089$.

16 a) Eberson, L.; Hartshorn, M. P.; Persson, O.; Radner, F. Chem. Commun. 1996, 2105 - 2112. b) Berkessel, A.; Adrio, J. A.; Huttenhain, D.; Neudorfl, J. J. Am. Chem. Soc. 2006, 128, 8421 - 8426.

${ }^{17}$ Kikuchi, O.; Hondo, Y.; Morihashi, K.; Nakayama, M. Bull. Chem. Soc. Jpn. 1988, 291 -292. 